\title{
Fatal Alcohol Poisonings and Gender Gap in Life Expectancy in Russia
}

\author{
Y. E. Razvodovsky \\ International Academy of Sobriety
}

\begin{abstract}
The difference in life expectancy between males and females in Russia is unprecedently high compared with developed countries.
\end{abstract}

Objective: This paper was design to estimate the aggregate level effect of binge drinking on the gender gap in life expectancy in Russian.

Method: Trends in male fatal alcohol poisoning rate (as a proxy for binge drinking) and gender gap in life expectancy from 1965 to 2015 were analyzed employing a distributed lags analysis in order to asses bivariate relationship between the two time series.

Results: The results of the time series analysis suggest a positive relation between fatal alcohol poisonings and gender gap in life expectancy $(r=0.85 ; S E=0.14)$.

Conclusions: The outcomes of this study provide indirect support for the hypothesis that binge drinking is a major contributor to the high gender gap in life expectancy and its dramatic fluctuations in Russia during the last few decades.

Keywords: Fatal alcohol poisonings, gender gap, life expectancy, Russia, 1965-2015.

\section{INTRODUCTION}

Life expectancy at birth defined as the average number of years that a newborn is expected to live if current mortality rates continue to apply ${ }^{[4]}$. Life expectancy is considered to be an important characteristic for quality of life ${ }^{[5,15]}$.

The difference in life expectancy between males and females in Russian Federation is unprecedently high compared with developed countries ${ }^{[1,3,5,6]}$. Russia was ranked first in the gender gap in life expectancy according to $\mathrm{WHO}^{[15,16]}$. The dramatic fluctuations in life expectancy in Russia during the last decades were one of the most significant developments in world health ${ }^{[7,12,16]}$. The wide gender gap in life expectancy in Russia is an extreme example of so-called "health-survival paradox" witch refers to the fact that women live longer than men, even though they report worse health. This phenomenon is usually attributed to a combination of biological, behavioral and socio-structural factors ${ }^{[11]}$. Men's higher engagement in risky behavior, higher vulnerability to stress, unhealthy lifestyle and reluctance to seek medical help in case of illness are among the most popular explanations of gender differences in life expectancy ${ }^{[4,11]}$.

There is strong evidence highlighting the key role of binge drinking in the phenomenon of strikingly higher gender gap in life expectancy and its dramatic fluctuations over the recent decades in Russia ${ }^{[5,6]}$. It has been reported that Russian men have considerably higher levels of alcohol consumption and alcohol-related mortality than women ${ }^{[7]}$. In line with this evidence we assume that devastating combination of high level of alcohol consumption per capita and binge drinking of vodka results in a close association between alcohol poisonings mortality rate (as an indicator of binge drinking) and gender gap in life expectancy at the aggregate level in Russia. This paper was design to estimate the aggregate level effect of binge drinking on gender gap in life expectancy in Russian.

\section{MATERiAl AND MethodS}

\subsection{Data}

The data on life expectancy and age-adjusted male alcohol poisonings mortality rate per 1000.000 of the population between 1965 and 2015 were taken from the Russian State Statistical Committee ( http://www.gks.ru). It should be noted that in Russia the fatal alcohol poisonings rate is likely to be a 
good measure of heavy episodic drinking ${ }^{[5,6]}$. Therefore, a common approach is to use the fatal alcohol poisonings rate as an indicator of binge drinking in this country ${ }^{[9]}$.

\subsection{Statistical Analysis}

To examine the relation between trends in alcohol poisonings mortality rate and gender gap in life expectancy across the study period a time series analysis was performed using the statistical package "Statistica 12. StatSoft." The dependent variable was the gender gap in life expectancy and the independent variable was male alcohol poisonings mortality rate. Bivariate correlations between the raw data from two time series can often be spurious due to common sources in the trends and due to autocorrelation ${ }^{[2]}$. One way to reduce the risk of obtaining a spurious relation between two variables that have common trends is to remove these trends by means of a "differencing" procedure.

The process whereby systematic variation within a time series is eliminated before the examination of potential causal relationships is referred to as "prewhitening." This is subsequently followed by an inspection of the cross-correlation function in order to estimate the association between the two prewhitened time series. It was Box and Jenkins ${ }^{[2]}$ who first proposed this particular method for undertaking a time series analysis between the time series. We used an unconstrained polynomial distributed lags analysis to estimate the relationship between the time series of fatal alcohol poisonings (independent variable) and gender gap in life expectancy (dependent variable) in this paper.

\subsection{Results}

Males and females show a gain in life expectancy of 0.1 and 3.0 years respectively between 1965 and 2015. The difference between life expectancy for men and women increased to 2.8 years over this period. Figure 1 shows the dynamic of life expectancy for both genders. Life expectancy is seen to decrease steadily for both genders until 1980 and than increased substantially between 1984 and 1986, with the gender gap fell to an all-time low of 9.5 years. By 1991, life expectancy had fallen for both genders and the decline accelerated through the years, reaching an all-time low of 57.4 years for men and of 71.1 years for women in 1994. Even though female life expectancy had fallen, the decline was not as steep as that of males. With male life expectancy dropped, the gender gap reached an alltime high of 13.7 years in 1994. There was a temporary improvement for both genders between 1995 and 1998, but since 1999, life expectancy turned downward again. Male and female life expectancy declined between 1999 and 2003, with male life expectancy dropping at a more rapid rate. Life expectancy for both genders increased substantially during the last decades, reaching an all-time high of 65.3 for men and of 76.5 for women respectively by 2014. However, the gender gap in life expectancy in Russia remains unprecedentedly high. The graphical evidence suggests that the temporal pattern of gender gap in life expectancy fits closely with changes in fatal alcohol poisonings (Figures 2).

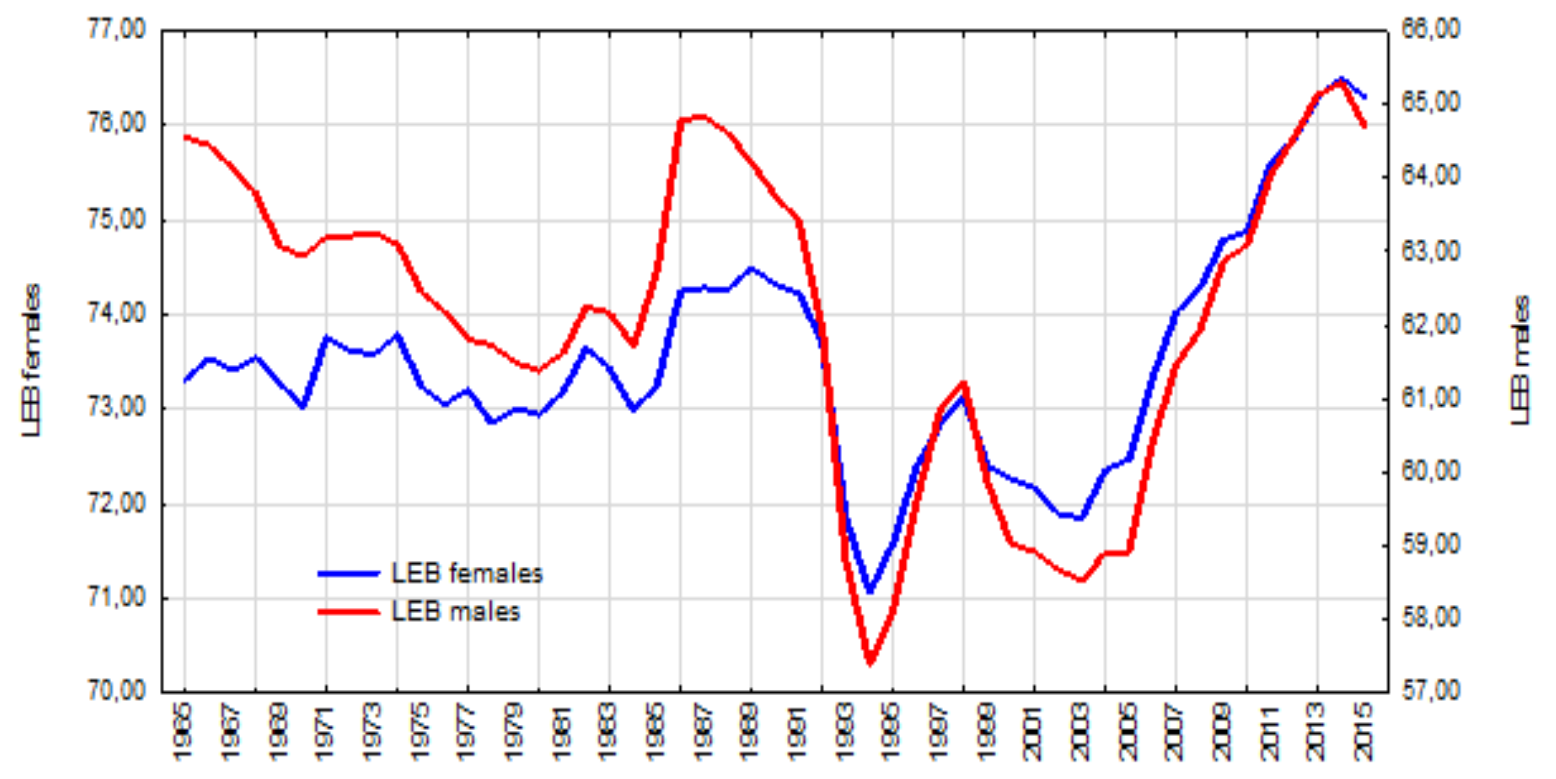

Figure1. Trends in male and female life expectancy at birth (LEB) in Russia between 1965 and 2015. 


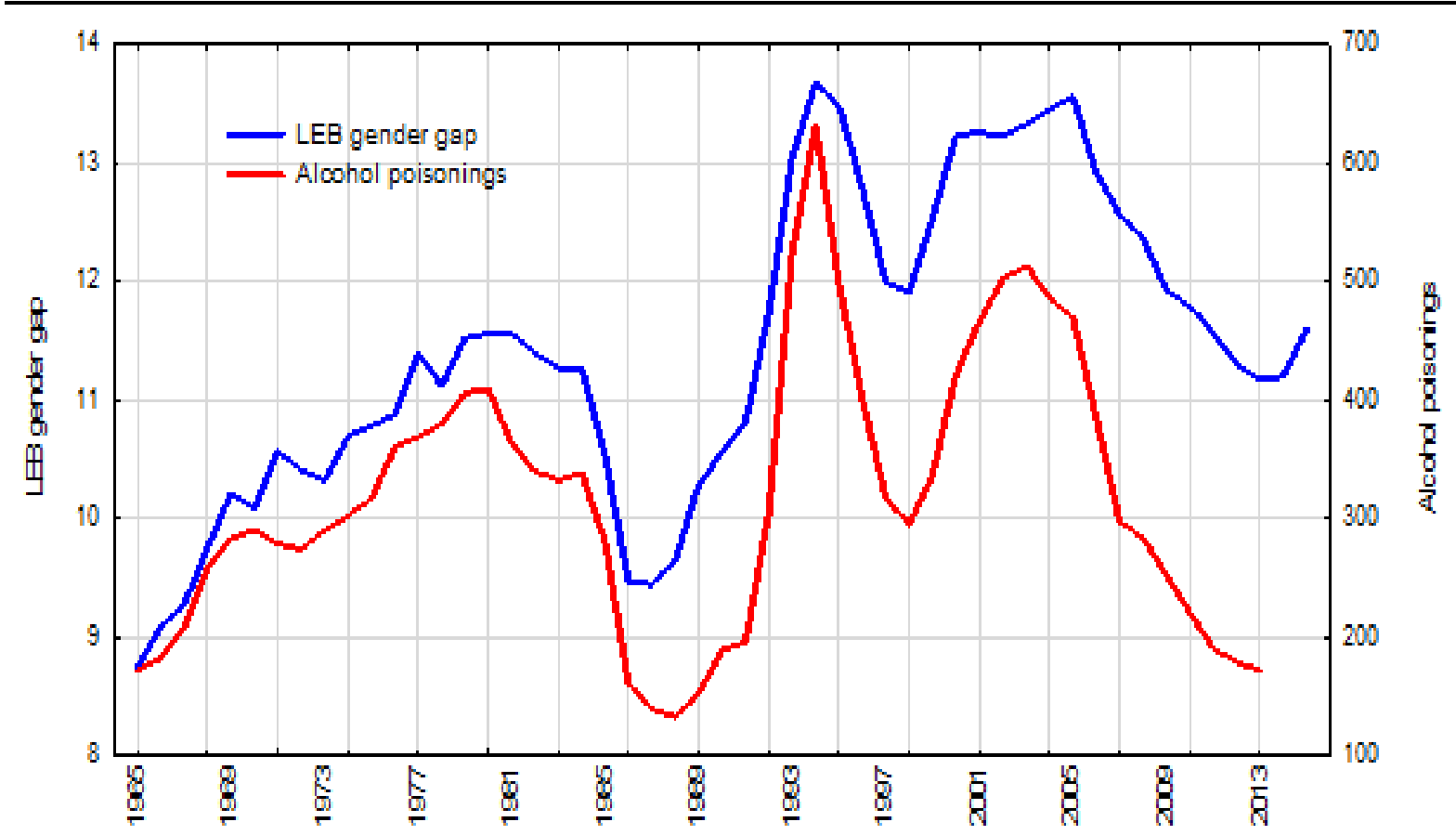

Figure2. Trends in the gender gap in life expectancy at birth (LEB) and alcohol poisonings mortality in Russia between 1965 and 2015.

A Spearman's rank correlation analysis suggests a strong association between the gender gap in life expectancy and alcohol poisonings mortality rate $(\mathrm{r}=0.79 ; \mathrm{p}<0,000)$. There were sharp trends in the time series data across the entire study period. These systematic variations were well accounted for by the application of first-order differencing and the specification of a first order moving average parameter (Figure 3). After pre-whitening the cross-correlations between fatal alcohol poisonings and gender gap in life expectancy time series were inspected (Figure 4). The outcome indicated statistically significant cross-correlation between the two variables at lag zero $(\mathrm{r}=0.85 ; \mathrm{SE}=0.14)$. The results of the distributed lags analysis suggest that only contemporaneous correlation (lag 0 ) is statistically significant $(\mathrm{r}=0.0059 ; \mathrm{p}=0.000)$.

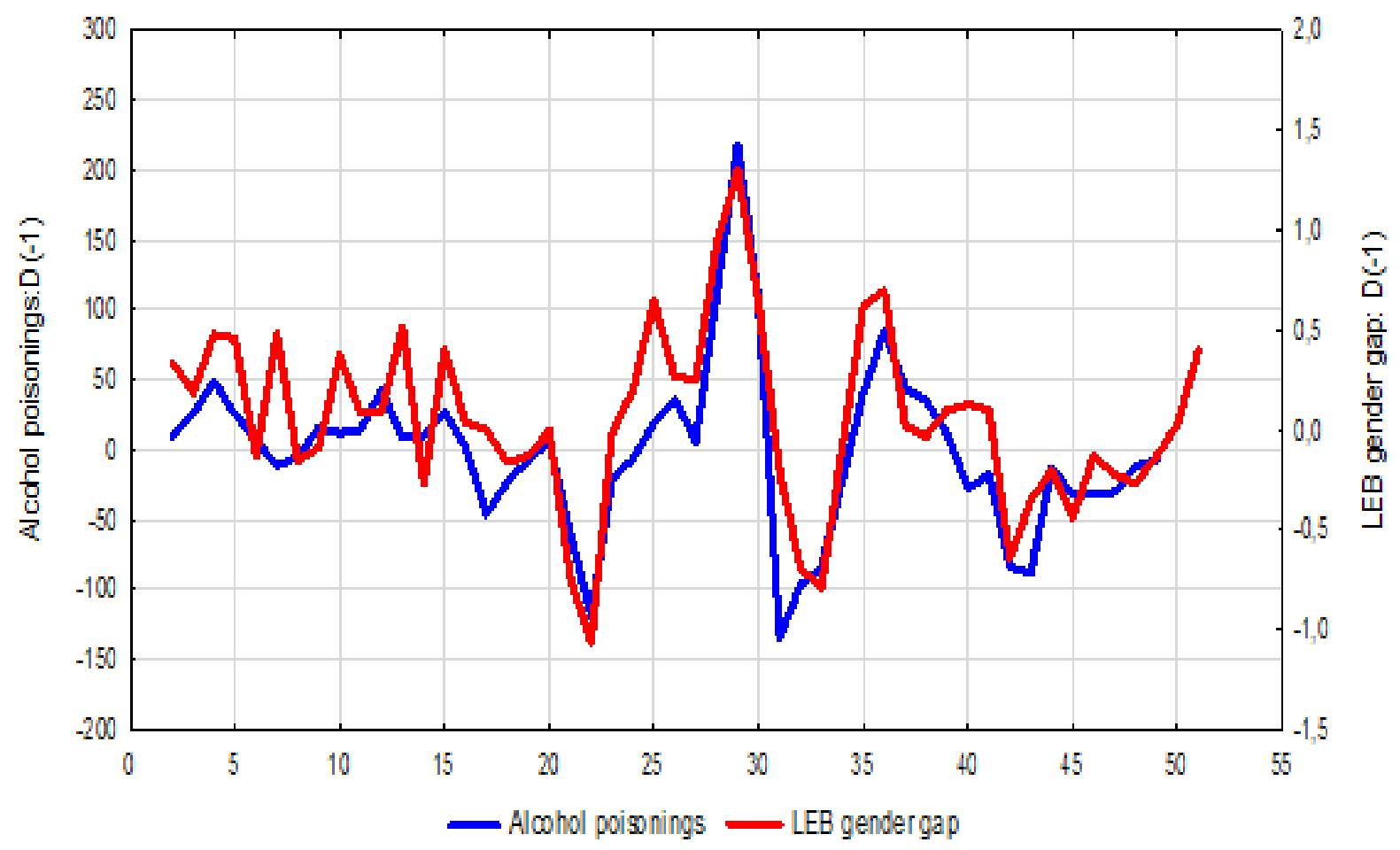

Figure3. Trends in the gender gap in life expectancy at birth (LEB) and alcohol poisonings mortality in Russia between 1956 and 2015 after differencing procedure. 


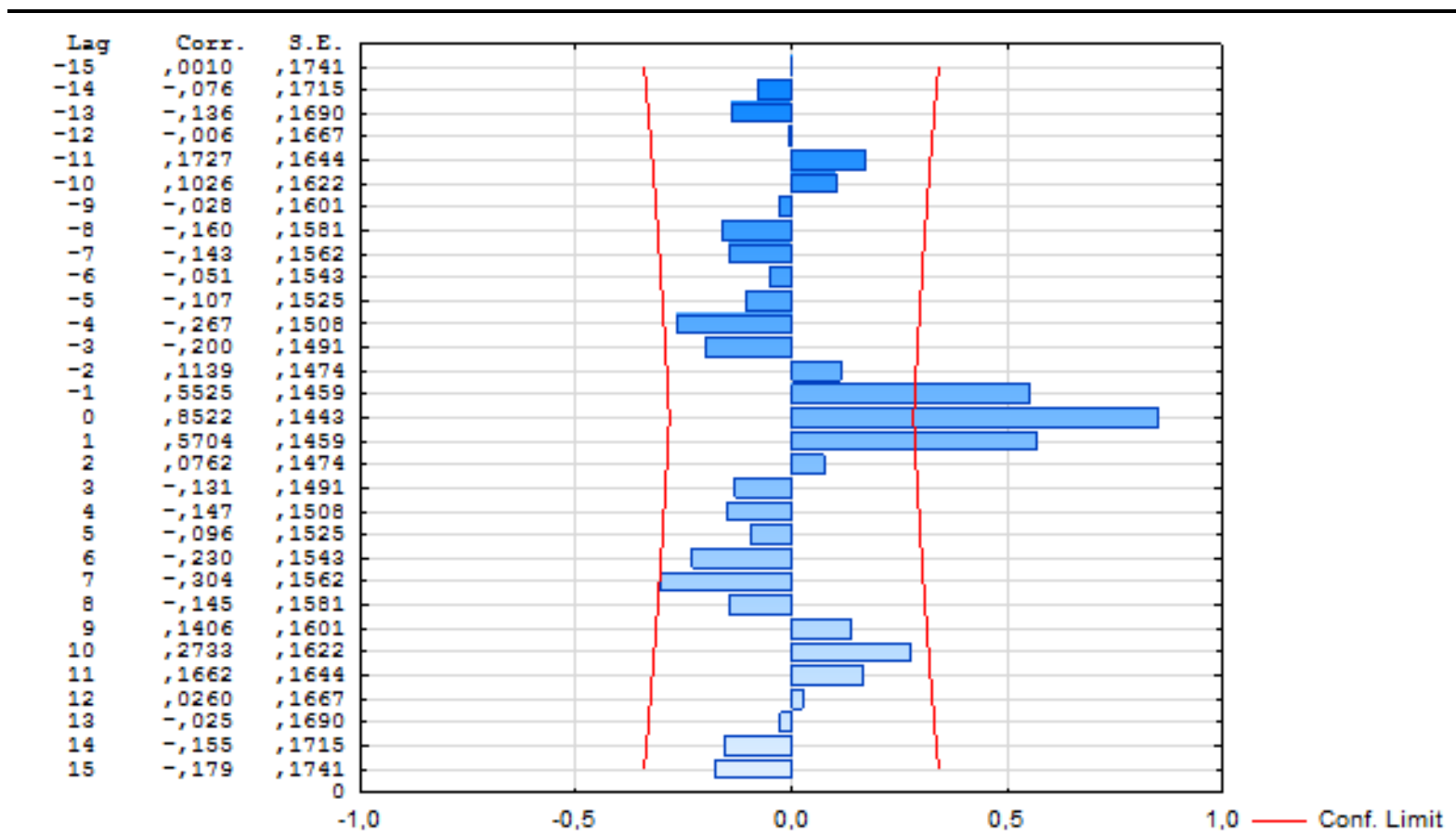

Figure4. Cross-correlation function.

\section{DISCUSSION}

The results of the time series analysis suggest a positive relation between fatal alcohol poisonings and gender gap in life expectancy and indirectly support the alcohol-related hypothesis that binge drinking accounts for extremely high gender gap in life expectancy and its dramatic fluctuations throughout the past 50 years in Russia. It should be recognized, however, that ignoring the confounding variables may imply that the effect of binge drinking on sex differences in life expectancy is overestimated.

It can be assumed that the effect of binge drinking on gender gap in life expectancy is a spurious indicator of the impact of other powerful risk factors such as smoking. There is general agreement that cigarette smoking is a major contributor to the sex difference in life expectancy in developed countries ${ }^{[4]}$. In European Union, for example, smoking accounts for $40-60 \%$ of the gender gap in mortality ${ }^{[16]}$. However, taking into account the fact that smoking has a long term effect on mortality, this factor alone cannot explain the trajectories in the gender gap in life expectancy in Russian during the last decades.

In conclusion, this study highlights key role of binge drinking in extremely high gender gap in life expectancy and its dramatic fluctuations in Russia during the last few decades. In relation to this, the Russian government needs to develop the effective alcohol control policy to address the high gender gap in life expectancy. Further research is needed to clarify more specifically the role of binge drinking in the difference between life expectancy for men and women in the Russian Federation.

\section{REFERENCES}

[1] Baykalova V. (2016). Life expectancy and gender gap in Russia: cross-regional variations. Budapest.

[2] Box GEP, Jenkins GM. (1976). Time Series Analysis: forecasting and control. London. HoldenDay Inc.

[3] Cockerman WC. (2013). The interaction of life expectancy and gender in a transitional state: the case of Russia. Sociology of Health \& Illnes. 34(6):943-957.

[4] Luy M, Minagava Y. (2014). Gender gaps - life expectancy and proportion of life in poor health. Health Reports. 25(12):12-19.

[5] Moskalewicz J, Razvodovsky Y, Wieczorek P. (2009). East-West disparities in alcohol-related harm within European Union. Paper presented at the KBS Annual Conference, Copenhagen, 1-5 June.

[6] Nemtsov AV, Razvodovsky YE (2016). Russian alcohol policy in false mirror. Alcohol Alcohol 4: 21 . 
[7] Nemtsov AV, Razvodovsky YE. (2016). Alcohol-related situation in Russia in the context of alcohol control policy. Sobriology. 4:66-74.

[8] Nemtsov AV, Razvodovsky YE. (2017). The estimation of the level of alcohol consumption in Russia: a review of the literature. Sobriology. 1:78-88.

[9] Nemtsov AV, Razvodovsky YE. Alcohol situation in Russia, 1980-2005. J Clin Psychiatry 2008:2; 52-60.

[10] Norström T. (1989). The use of aggregate data in alcohol epidemiology. British Journal of Addiction. 84:969-977.

[11] Oksuzyan A, Juel K, Vaupel1 JW, Christensen K. (2008). Men: good health and high mortality. Sex differences in health and aging. Aging Clin Exp Res. 20(2): 91-102.

[12] Razvodovsky YE, Nemtsov AV. (2016). Alcohol-related component of the mortality decline in Russia after 2003. The Questions of Narcology. 3:63-70.

[13] Razvodovsky YE. (2009). Aggregate level beverage specific effect of alcohol sale on myocardial infarction mortality rate. Adicciones, 21(3), 229-238.

[14] Razvodovsky YE. (2013). Estimation of the level of alcohol consumption in Russia. ICAP Periodic Review Drinking and Culture 8: 6-10.

[15] Reamy J., Oreskoviae S. Life expectancy in Central and Eastern European countries and Newly Independent States of the former Soviet Union. Croatian Medical Journal. 1999.40(2):1-6.

[16] Van Oyen H, Nusselder W, Jagger C, Kolip P, Cambois E, Robine JM. (2013). Gender differences in healthy life years within the EU: an exploration of the "health-survival" paradox. Int J Public Health. 58:143-155.

Citation: Y. E., Razvodovsky. "Fatal Alcohol Poisonings and Gender Gap in Life Expectancy in Russia." International Journal of Research in Sociology and Anthropology (IJRSA), vol 3, no. 3, 2017, pp. 1-5. doi:DOI: http://dx.doi.org/10.20431/2454-8677.0303001

Copyright: (๑) 2017 Authors. This is an open-access article distributed under the terms of the Creative Commons Attribution License, which permits unrestricted use, distribution, and reproduction in any medium, provided the original author and source are credited. 